\title{
Altered immunosuppressive activity of alveolar macrophages in farmer's lung disease
}

\author{
A. Dakhama, E. Israël-Assayag, Y. Cormier
}

\begin{abstract}
Altered immunosuppressive activity of alveolar macrophages in farmer's lung disease. A. Dakhama, E. Israël-Assayag, Y. Cormier. (CERS Journals Ltd 1996.

ABSTRACT: Since normal alveolar macrophages (AMs) can suppress T-cell proliferation to mitogenic and antigenic stimuli both in vitro and in vivo, we questioned whether an altered AM immunosuppressive activity could account for the alveolar lymphocytosis observed in farmer's lung (FL) and whether granulocyte/macrophage colony-stimulating factor (GM-CSF), a cytokine able to abrogate AM-induced immunosuppression, is involved in the process.

The ability of different concentrations of AMs to inhibit lymphocyte proliferation in response to the T-cell-specific mitogen phytohaemagglutin (PHA) after in vitro culture was tested in three groups of subjects: 12 patients with FL; four asymptomatic farmers (AS); and six normal volunteers (N). Release of GM-CSF by AMs was also measured.

At all ratios tested, AMs from patients with FL did not suppress the lymphoproliferation but instead had an enhancing effect. In AS, AMs enhanced the proliferation at a lower ratio but inhibited it at high ratios. In $\mathrm{N}$ subjects, as described previously, AMs increasingly inhibited the blastogenesis of lymphocytes $(\mathrm{L})$ at increasing ratios of AM:L. In some patients with FL, AMs spontaneously released more GM-CSF than in normal volunteers $\left(206 \pm 84\right.$ versus $29 \pm 14 \mathrm{pg} \cdot \mathrm{mL}^{-1}$, respectively). In AS, GM-CSF release was intermediate $\left(74 \pm 36 \mathrm{pg} \cdot \mathrm{mL}^{-1}\right)$.

In conclusion, a defect in the ability of alveolar macrophages to suppress the proliferation of lymphocytes in the lung of patients with farmer's lung is a major factor accounting for the development of the observed lymphocytic alveolitis. Granulocyte/macrophage colony-stimulating factor could be one factor which may contribute to this alteration.
\end{abstract}

Eur Respir J., 1996, 9, 1456-1462.

Unité de Recherche, Centre de Pneumologie de l'Hôpital Laval, Université Laval, SainteFoy (Québec), Canada.

Correspondence: Y. Cormier

Centre de Pneumologie

Hôpital Laval

2725 Chemin Sainte-Foy

Sainte-Foy

Québec

Canada G1V 4G5

Keywords: Alveolar macrophages hypersensitivity pneumonitis interstitial lung disease

Received: October 301995

Accepted after revision March 291996

Supported by the Respiratory Health Network of Centres of Excellence, Canada.
Farmer's lung (FL) is a type of hypersensitivity pneumonitis characterized by a massive proliferation of lymphocytes, mostly of the cytotoxic/suppressor subtype $(\mathrm{CD} 8+)$, which accumulate within the lung parenchyma and alveolar space of sensitized individuals, and may mediate tissue damage [1-3]. The resulting alveolar lymphocytosis can persist after an acute episode of the disease in subjects who are still exposed to the offending antigens that occur in the farm environment [4, 5]. A milder lymphocytosis can be observed in asymptomatic but exposed dairy farmers [6]. A recent investigation suggested that both acute and persisting alveolar lymphocytosis are sustained by an in vivo production of interleukin (IL)-2 [7]. Different hypotheses to explain why only a proportion of exposed individuals develop the symptomatic disease include: a massive exposure to the antigens [8]; a viral infection [9]; or a co-exposure [10]. However, direct data in humans supporting such concepts are not yet available. Individual susceptibility may be an important predisposing factor to the disease; however, some studies have failed to detect genetic markers for FL [11]. We postulated that changes in host cell functions could be important in the development of symptomatic FL.

In normal lung, where they constitute the majority of the alveolar cell population, alveolar macrophages (AMs) have the ability to suppress antigenic and mitogenic [1214], as well as IL-2-induced lymphocyte proliferation [15]. AMs can also suppress T-cell cytotoxicity [16], and immunoglobulin production [17, 18]. Recent studies demonstrated that AMs selectively inhibit the proliferation of T-cells without affecting their initial activation and expression of effector functions $[19,20]$. Thus, by limiting the overall expansion of activated T-cells in vivo, this immunosuppressive activity of AMs would be necessary to protect against T-cell-mediated lung tissue damage that may occur in response to repeated inhalations of nonpathogenic antigens [21]. A previous study [22] showed that AM-induced T-cell suppression is reversible by granulocyte/macrophage colony-stimulating factor (GM-CSF). GM-CSF is secreted by airway epithelial cells and macrophages upon stimulation by antigen or cytokines, such as IL-1 and tumour necrosis factor- $\alpha$ $(\mathrm{TNF}-\alpha)$ [23]. Moreover, GM-CSF may contribute to the proliferation of AMs and the recruitment of lymphocytes [24].

In FL, AMs are actively involved in the disease and release proinflammatory mediators and cytokines [25, 26]. Although their absolute number is increased in the bronchoalveolar lavage (BAL) of patients with acute FL, 
their relative proportion is considerably diminished whilst lymphocytes become the predominant alveolar cell population [27]. It thus appears feasible that an alteration (downregulation) of the immunosuppressive activity of AMs is likely to occur in FL, therefore allowing for the development of the lymphocytic alveolitis. To our knowledge, no study has reported the lymphosuppressive function of AMs from patients with FL or any other form of hypersensitivity pneumonitis.

To test this hypothesis, we evaluated the ability of AMs to suppress autologous lymphocyte proliferation in vitro in three groups of subjects (patients with FL, asymptomatic farmers, and normal nonfarming volunteers). The results show that the immunosuppressive activity of AMs is markedly altered in patients with acute FL, and to a lesser degree in asymptomatic farmers with a lymphocytic alveolitis. We also measured the amounts of GM-CSF produced spontaneously by AMs cultured in vitro for 24 h. A positive correlation was found between amounts of GM-CSF secreted by macrophages and the percentage of proliferation of AM-peripheral blood mononuclear cell (PBMC) co-cultures. This may be one of the mechanisms involved in the alteration of the normal suppressive AM activity in patients with acute FL.

\section{Materials and methods}

\section{Subjects}

The study included 12 patients with FL, four asymptomatic farmers with a lymphocytic alveolitis (AS), and six normal nonfarming controls $(\mathrm{N})$. The diagnosis of FL was based on the criteria of CHEMLIK et al. [28]. All patients were dairy farmers and had a clinical history suggestive of FL, including inspiratory crackles on physical examination, interstitial infiltration on chest radiographs, and altered lung functions. All subjects were nonsmokers, and none was receiving immunosuppressive therapy at the time of this study. BAL and venous blood were obtained from all patients as a part of their clinical evaluation and from other subjects on written consent.

\section{Bronchoalveolar lavage}

BAL was performed using a fibreoptic bronchoscope as described previously [4]. In brief, a total volume of $300 \mathrm{~mL}$ of sterile nonpyrogenic saline was instilled in $60 \mathrm{~mL}$ aliquots into a wedged segment, usually of the right middle lobe. After each instillation, the fluid was gently aspirated, placed in $50 \mathrm{~mL}$ centrifugation tubes and kept on ice until processed.

\section{Preparation of alveolar macrophages (AM)}

Cells were separated from BAL fluids by centrifugation at $400 \times \mathrm{g}$ for $10 \mathrm{~min}$ at $4^{\circ} \mathrm{C}$. After two successive washes with Hank's balanced salt solution (HBSS), the cells were counted on a haemacytometer and resuspended at $10^{7}$ cells $\cdot \mathrm{mL}^{-1}$ in RPMI-1640 (Roswell Park Memorial Institute-1640) medium (Gibco-Canada) supplemented with $10 \%$ heat-inactivated foetal bovine serum and $1 \%$ penicillin-streptomycin (complete medium). Differential cell counts were performed on Diff-Quik ${ }^{\circledR}$ and esterasestained cytocentrifuge preparations.

AMs were purified by adherence on sterile plastic dishes for $1 \mathrm{~h}$ at $37^{\circ} \mathrm{C}$ in a $5 \% \mathrm{CO}_{2}$-enriched humidified atmosphere. After removal of nonadherent cells by several washes with HBSS, the adherent cells were incubated at $4{ }^{\circ} \mathrm{C}$ in ice-cold $\mathrm{HBSS}$ for $30 \mathrm{~min}$, and finally detached by gently scraping with a sterile rubber policeman. Typically, this method yielded 90-95\% AM-enriched cell preparation as indicated by esterase staining. Cell viability, as estimated by trypan blue exclusion, was above $97 \%$.

\section{Preparation of PBMC}

Heparinized blood was obtained from all patients and controls. PBMC were separated from whole blood by Ficoll-paque density gradient centrifugation, washed three times in HBSS and resuspended in complete RPMI at $0.5 \times 10^{6}$ cells $\cdot \mathrm{mL}^{-1}$. These cells were more than $97 \%$ viable. Whole PBMC were used in co-cultures in order for blood monocytes to provide the accessory function for the lymphocyte response to phytohaemagglutin (PHA).

\section{Preparation of blood monocytes}

Monocytes were prepared according to a method described previously [29]. Briefly, PBMC were allowed to adhere to serum precoated plastic dishes for $1 \mathrm{~h}$ at $37^{\circ} \mathrm{C}$. Nonadherent cells were removed by washing. Adherent cells were covered with ice-cold HBSS and incubated at $4^{\circ} \mathrm{C}$ for $30 \mathrm{~min}$. The cells were removed by gentle scraping with a rubber policeman and resuspended at various concentrations in RPMI medium. These cells were found to be $90-95 \%$ positive for esterase.

\section{In vitro assays for immunosuppressive activity of $A M s$}

Purified AMs, or in some cases monocytes, were co-cultured for $72 \mathrm{~h}$ with autologous PBMC at various ratios $(0.5: 1,1: 1,2: 1)$ in the presence or absence of PHA (1 $\left.\mu \mathrm{g} \cdot \mathrm{mL}^{-1}\right)$ in 96 -well microtitre plates, each well containing $200 \mu \mathrm{L}$ of complete medium and $5 \times 10^{4}$ PBMC. Similar co-cultures were also obtained at a $2: 1$ ratio of monocytes:PBMC, i.e. a triple concentration of monocytes. The cultures were pulsed with $1 \mu \mathrm{Ci}$ tritiated-thymidine for the last $12 \mathrm{~h}$. The cells were then harvested onto fibreglass filters and the radioactivity counted in a Packard matrix $\beta$ counter. The data were obtained as mean counts per minute (cpm) from quadruplicate values after subtracting the background from unstimulated cultures, and the results were expressed as a percentage of PHA-stimulated PBMC without AMs.

\section{Detection of spontaneous release of GM-CSF}

GM-CSF was measured by enzyme-linked immunosorbent assay (ELISA) (PerSeptive Diagnosis, Cambridge, MA, USA) in BAL fluid and in the supernatants from AMs after $24 \mathrm{~h}$ of culture at $5 \times 105$ cells $\cdot \mathrm{mL}^{-1}$ in complete RPMI medium. 


\section{Statistical analysis}

For graphical presentation of data, results of representative measures were expressed as mean \pm SEM. For comparisons between group means, a one-way analysis of variance (ANOVA) was performed. Normality and homogeneity of variances was verified to validate tests. When a significant overall difference was observed, pairwise differences between group means were evaluated using Scheffe's comparison. Pearson's correlation coefficient was used for the relationship between GM-CSF and lymphoproliferation. The statistical software package Statistical Analysis System (SAS) was used for all analyses.

\section{Results}

Although the percentage of macrophages was considerably decreased in the BAL from patients with FL (fig. 1a), there was a significant increase in their absolute number with respect to total cells when compared to normal control values: $201 \pm 18 \times 10^{3}$ versus $59 \pm 12 \times 10^{3}$

a)

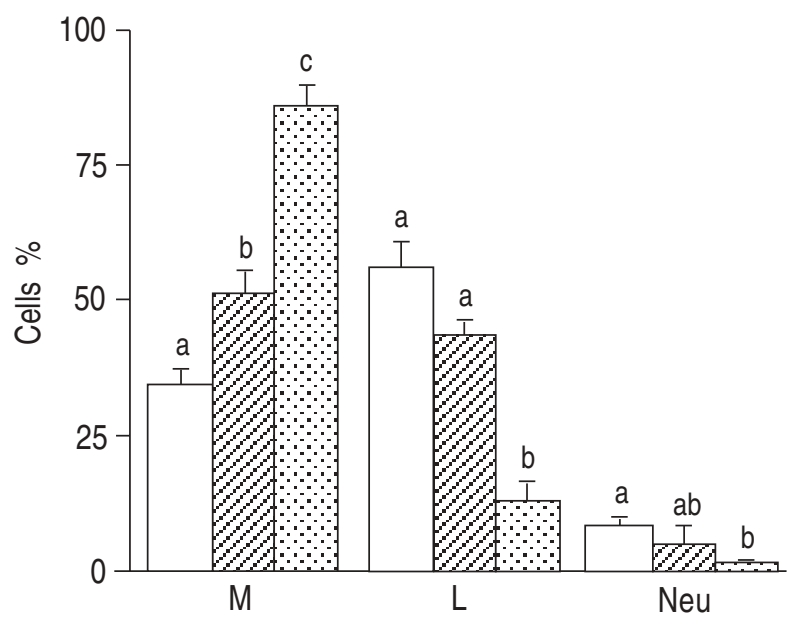

b)

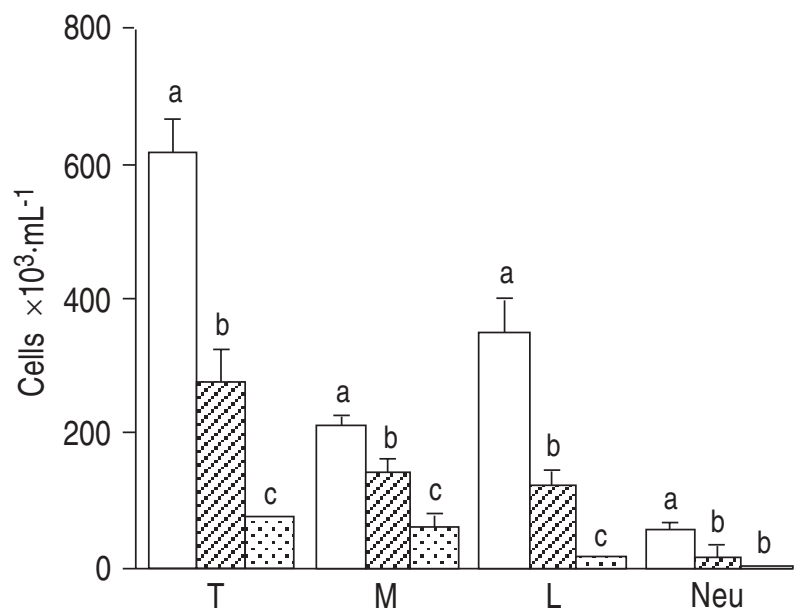

Fig. 1. - a) Bronchoalveolar lavage (BAL) differential cell counts, in percentage of total cells; b) BAL total cell counts in number of cells $\mathrm{mL}^{-1}$. Values are presented as mean \pm SEM. For total cells and each cell type, bars with different letters are significantly different $(\mathrm{p}<0.05)$ between groups of subjects. T: total cells; M: macrophages; L: lymphocytes; Neu: neutrophils; $\square$ : patients with farmer's lung ( $\mathrm{n}=12)$; $\square$ : asy-mptomatic farmers $(n=4) ; \square$ : normal non-farmer volunteers $(\mathrm{n}=6)$. macrophages $\cdot \mathrm{mL}$ or recovered fluid, respectively $(\mathrm{p}<0.05)$ (fig. 1b). BAL from asymptomatics showed intermediate values for macrophage counts. A significant increase in neutrophil numbers was observed only in the FL group.

The proliferation responses of PBMC to PHA after in vitro culture in the presence of increasing proportions of autologous AM are presented in figure 2. Results are given in percent proliferation of PHA-stimulated PBMC without AM (100\%). As expected, AM from normal subjects suppressed PHA-induced proliferation of autologous PBMC. This suppression increased with increasing AM:PBMC ratios. $\mathrm{P}$ values for ratios $0.5: 1,1: 1$, and 2 were respectively: $0.101,0.023$ and 0.002 . In asymptomatic farmers, AM at low ratio (0.5:1) did not suppress but stimulated lymphoproliferation to PHA ( $\mathrm{p}=0.001)$. However, when higher concentrations of AM were added, a normal suppressive activity was restored (ratio 2:1, $\mathrm{p}=0.023$ ). AM from patients with FL failed to suppress PBMC proliferation at any ratio of AM:PBMC tested. A significant enhancing effect was seen at ratios $0.5: 1$ and $1: 1(p=0.002$ and 0.023$)$. At the $2: 1$ ratio, proliferation was not different than control cultures $(p=0.55)$. Comparison between groups shows that AM from normal subjects had significantly higher suppressive activity at all ratios tested than AM from subjects with acute $\mathrm{FL}(\mathrm{p}<0.01)$. AM from asymptomatic farmers had intermediate effects. At low ratios they failed to suppress PBMC proliferation, but at higher ratios their suppressive function was significantly greater than that of AM from the subjects with acute FL $(p<0.01)$. AS had more suppression at higher AM:PBMC ratios. Figure 3 shows the results of an experiment where additional purified blood monocytes were added in the cultures. When AM were substituted by monocytes, no suppression was observed, whereas

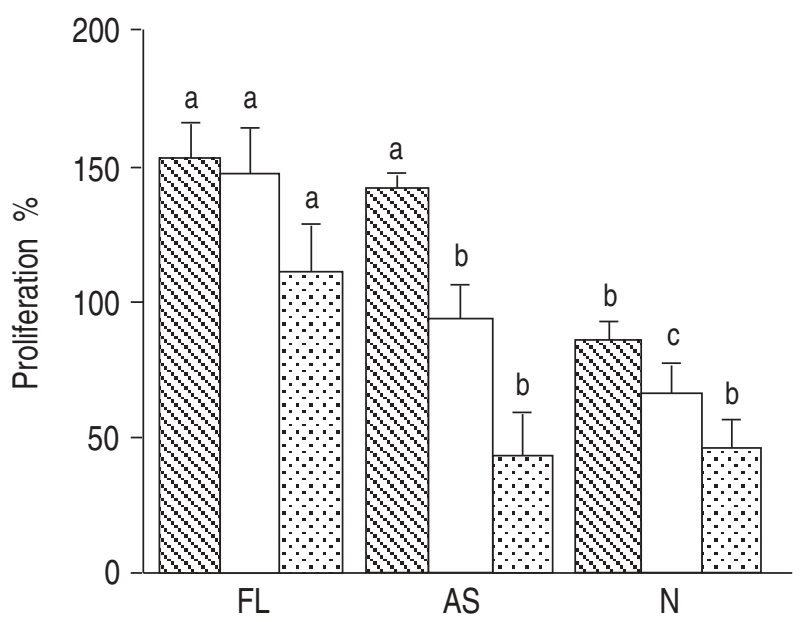

Fig. 2. - Phytohaemagglutinin-induced proliferation of lymphocytes of peripheral blood mononuclear cells (PBMC) in the presence of increasing concentrations of autologous alveolar macrophages. Values are presented as mean \pm SEM. The basal counts for unstimulated PBMC with or without AMs at each ratio were negligible and not different within or between the groups. Results are expressed as percentage of PHA-induced PBMC response (without $\mathrm{AM}=100 \%$ ). Values with different letters are significantly different between groups $(\mathrm{p}<0.01)$. AMs from FL patients significantly stimulated PBMC proliferation, while those from normal subjects suppressed this activity. AMs from asymptomatics farmers were able to suppress PBMC proliferation only at high AM:PBMC ratios. PHA: phytohaemagglutinin; AMs: alveolar macrophages; FL: farmer's lung. For further definitions see legend to figure 1. $\square$ : 0.5:1 (AM:PBMC); $\square: 1: 1$ (AM:PBMC); $\square$ : 2:1 (AM:PBMC). 
a) FL subject

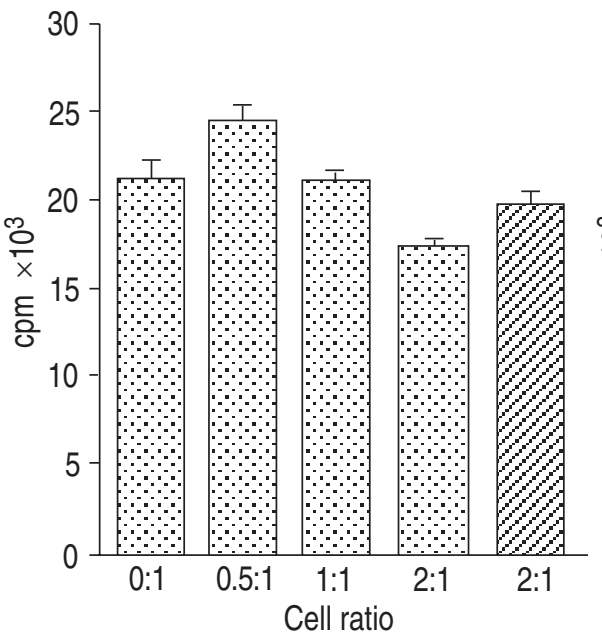

b) AS subject

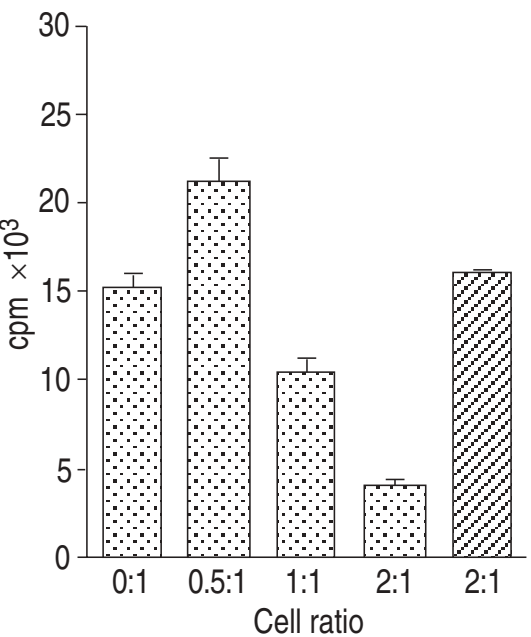

c) N subject

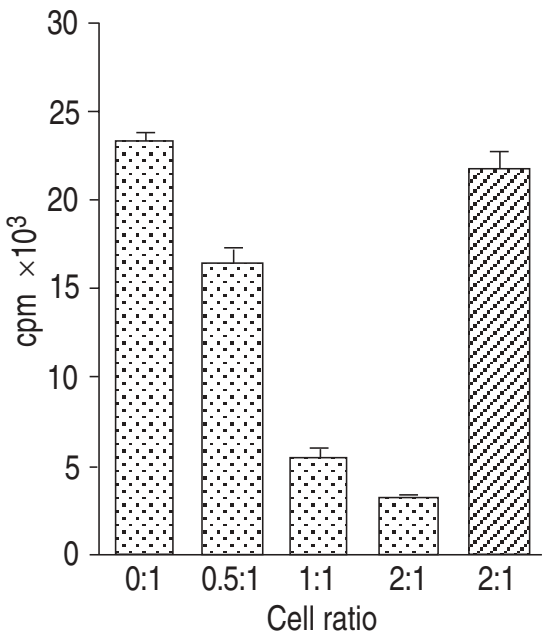

Fig. 3. - Phytohaemagglutinin-induced proliferation responses of triated-thymidine labelled lymphocytes of peripheral blood mononuclear cells (PBMC) in the presence of increasing concentrations of autologous alveolar macrophages (AMs) or purified blood monocytes (Mono), for one typical subject from each group: a) patient with farmer's lung (FL); b) asymptomatic farmer (AS); c) normal nonfarming volunteer (N). Values are presented as mean \pm SEM. The persisting proliferative response when AMs were substituted by monocytes confirms that the suppression observed was not due to cell crowding. $\square$ : AM:PBMC; $\square$ : Mono:PBMC; cpm: counts per minute.

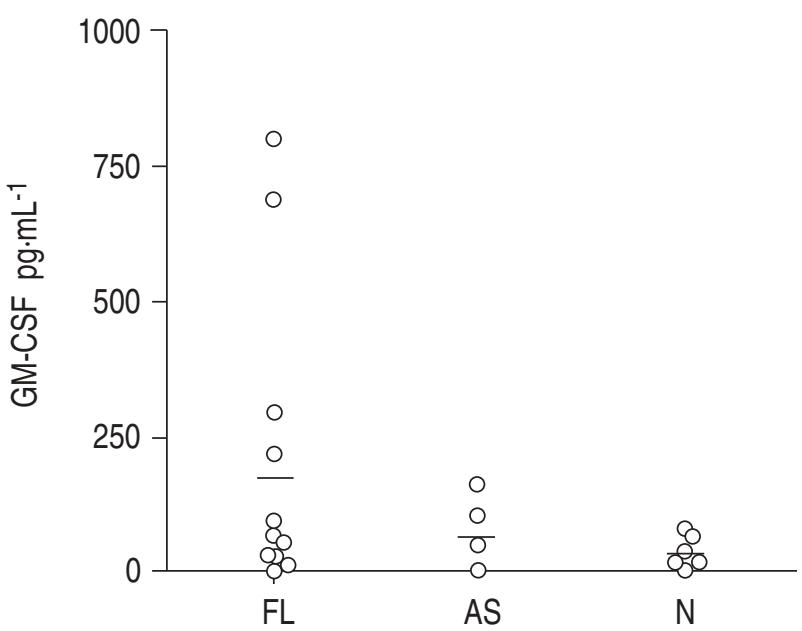

Fig. 4. - Spontaneous in vitro release of granulocyte/macrophage colony-stimulating factor (GM-CSF) by alveolar macrophages from the three groups of subjects. Although four subjects in the FL group had higher levels of GM-CSF, the difference between groups did not reach statistical significance. Horizontal bar indictes mean value. For further definitions see legend to figure 1 .

in $\mathrm{N}$ and $\mathrm{AS}$, increasing concentration of $\mathrm{AM}$ induced an increased suppression.

No GM-CSF was found in the BAL fluids. Figure 4 illustrates the values of spontaneous release of GM-CSF from AMs in the three groups of subjects. AMs from patients with acute FL released more GM-CSF (206 \pm 84 $\left.\mathrm{pg} \cdot \mathrm{mL}^{-1}\right)$ than AMs from asymptomatic farmers $(74 \pm 36$ $\left.\mathrm{pg} \cdot \mathrm{mL}^{-1}\right)$ or normal volunteers $\left(29 \pm 14 \mathrm{pg} \cdot \mathrm{mL}^{-1}\right)$. Although the differences between the groups did not reach statistical significance, AMs from some patients, but not all, spontaneously released substantial amounts of GM-CSF after in vitro culture. At all ratios tested, a positive correlation was found between the proliferation index and amounts of GM-CSF secreted by AMs (at ratio 0.5:1, $\mathrm{r}=0.666$ and $\mathrm{p}=0.0013$; at ratio $1: 1, \mathrm{r}=0.727$ and $\mathrm{p}=0.0001$; at ratio $2: 1, r=0.779$ and $p=0.0001$, for all subjects). Figure 5 illustrates this correlation at ratio $2: 1$.

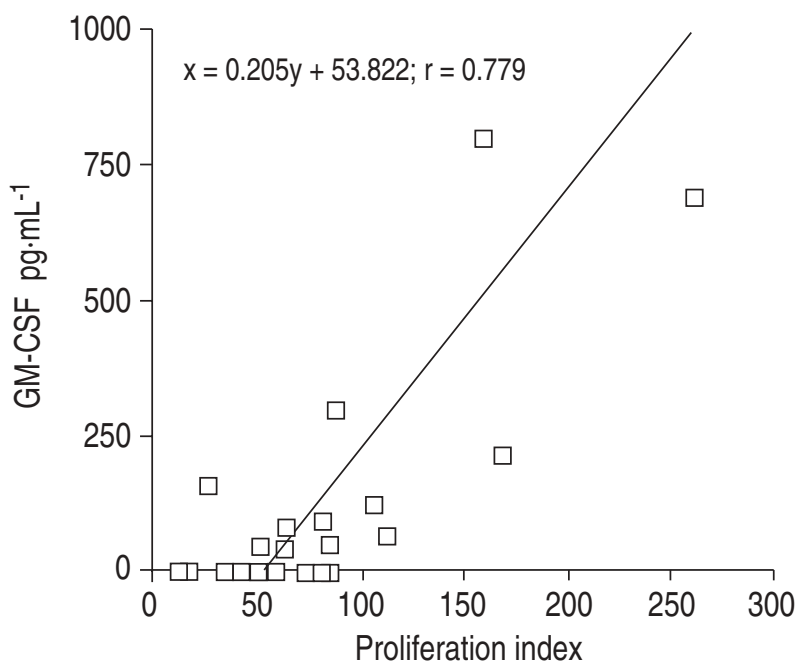

Fig. 5. - Correlation between amounts of granulocyte/macrophage colony-stimulating factor (GM-CSF) produced by in vitro cultures of alveolar macrophages and percentage of lymphoproliferation in AM:PBMC 2:1 ratio co-cultures for all subjects. AM: alveolar macrophage; PBMC: peripheral blood mononuclear cell.

\section{Discussion}

The present investigation focused on the immunosuppressive activity of AMs as a potential candidate which may be involved in the development of the lymphocytic alveolitis observed in FL disease. The results clearly demonstrate a defect in the ability of AMs from patients with FL to suppress in vitro mitogenic proliferation of autologous lymphocytes. The development of this downregulation of the immunosuppressive activity is probably progressive, as shown by a lesser degree of alteration in asymptomatic farmers. Since this immunosuppressive activity may play a role in protecting the lung against excessive lymphocyte accumulation and subsequent tissue damage in response to continuous antigenic challenges [21], such an altered activity may be of significance 
in the pathogenesis of FL. Such an alteration is even more important when one considers that the in vivo ratio of AM:lymphocyte (as estimated by BAL) is significantly decreased in the lung of patients with FL and in asymptomatic farmers with a lymphocytic alveolitis $(0.6$ and 1.3, respectively) compared to normal subjects (6.8).

Although IL-2 may be one mechanism which contributes to lymphocyte accumulation in the lung of patients with FL [7, 30], since this cytokine does not inhibit the suppressive effect of AMs [31] it cannot be held responsible for the altered AM immunosuppressive activity observed in this study.

A previous study has shown that cigarette smoke does not alter the immunosuppressive activity of AMs in normal subjects [32]. This observation is of great interest, since farmers who smoke are less likely to develop precipitating antibodies to the antigens responsible for farmer's lung and have a lower incidence of FL than nonsmokers [33]. In sarcoidosis, another disease where large numbers of lymphocytes accumulate in the lung parenchyma, there are conflicting data on the maintenance or alteration of this AM activity [34, 35].

The mechanisms by which AMs achieve this lymphosuppression are still incompletely characterized. It is generally admitted that this suppression primarily requires cell-to-cell contact [29, 31,34], although some mediators of inflammation, such as reactive oxygen species and prostaglandins [15], and the transforming growth factor- $\beta_{1}$ (TGF- $\beta_{1}$ ) may also contribute to this effect [36, 37]. A recent study suggested that suppression of CD3induced T-cell signal transduction by normal AMs may be mediated by surfactant protein-A (SP-A) [38]. In this study, in contrast to normal AMs, the amount of membrane-associated SP-A was substantially decreased in AMs from patients with interstitial lung disease where the suppressive activity was altered. Whether a normal immunosuppressive activity may be restored by addition of surfactant components needs further study. Other workers [22] have demonstrated that pretreatment of mouse AMs with GM-CSF (either recombinant form or natural form generated in lung-conditioned medium following exposure to lipopolysaccharide) abrogated mitogen-induced T-cell proliferation. In our study, we attempted to measure GM-CSF in vivo in concentrated BAL fluids from our subjects but failed to detect this cytokine, whilst substantial amounts were spontaneously released from AMs in vitro. This failure could be explained by the possible instability of this cytokine in the presence of a variety of proteolytic enzymes that might be recovered in the BAL fluids under such disease conditions. Apart from lymphocytes and macrophages, bronchial epithelial cells [39] and mast cells [40] may constitute other potential sources for GM-CSF in vivo. There is evidence that mast cells are increased in numbers in the BAL of patients with FL [41], however their exact role in the disease remains undefined. A more recent investigation successfully demonstrated GM-CSF by immunocytochemistry on mast cells and macrophages in the lung parenchyma of patients with FL [42].

Of particular interest in our study is the finding that AMs from patients with FL enhance, rather than suppress, the mitogenic response of autologous lymphocytes. This duality in AM function is likely to be reflected by the heterogeneity of this cell population. In FL, AMs are significantly increased in total numbers not only during the acute phase of the disease but also in asymptomatics. Compared to normal control values, in our study there were approximately threefold and twofold increases in this cell population in patients with FL and in asymptomatics, respectively. It is possible that such an increase might compensate for the normal immunosuppressive activity by diluting the local macrophage population. However, the stimulatory effect of these new macrophages adds another dimension to the alteration of the suppressive activity observed in this study. Whether this increase in AM population results from an in situ proliferation of a specific "subpopulation" of resident AMs or represents a pool of newly recruited monocyte-like macrophages need to be determined. Some recent observations may suggest the former possibility [43]; however, the lower proliferation indices reported may not account for the total increase observed in the present study. It is known that AMs can be induced to proliferate when stimulated by GM-CSF [44]. In addition, actively proliferating macrophages could be demonstrated in the peritoneum of transgenic mice for GM-CSF (45). In the present study, increased production of GM-CSF by AMs from patients with the active disease was detected and correlated with an increase in lymphoproliferation. Since this induction of AM proliferation might be important in the pathogenesis of FL, a possible involvement of IL-2 could not be totally excluded, since IL-2 receptor is more predominantly expressed on AMs in patients with FL [46]. Further studies are required to better characterize the stimulatory mechanism of these AMs and to determine whether an induction of AM proliferation would interfere with the immunosuppressive function. Other cytokines, with anti-inflammatory properties, such as TGF- $\beta$ and IL-10, may also be involved in the disease. It would be interesting to look at the participation of these cytokines in different phases of FL in subsequent studies.

Based on the present observations, with the assumption that a heterogeneity in the alveolar macrophage population may also reflect a heterogeneity in alveolar macrophage function, we speculate that the prevalence of a specific "subpopulation" of alveolar macrophages (suppressive or stimulatory) might determine the normal immunological homeostasis or the disease condition. Better understanding of these regulatory mechanisms will help us determine why only some exposed individuals develop farmer's lung disease. Promoting the natural immunosuppression could be a promising avenue for the treatment of this disease.

\section{References}

1. Fink JH. Hypersensitivity pneumonitis. J Allergy Clin Immunol 1984; 74: 1-9.

2. Semenzato G, Trentin L, Zambello R, Agostini C, Cipriani A, Marcer G. Different types of cytotoxic lymphocytes recovered from the lungs of patients with hypersensitivity pneumonitis. Am J Respir Crit Care Med 1988; 137: 70-81.

3. Salvaggio JE. Recent advances in pathogenesis of allergic alveolitis. Clin Exp Allergy 1990; 20: 137-144.

4. Cormier Y, Bélanger J, Laviolette M. Persistent bronchoalveolar lymphocytosis in asymptomatic farmers. $\mathrm{Am}$ Rev Respir Dis 1986; 133: 843-847. 
5. Cormier Y, Bélanger J, Laviolette M. Prognostic significance of bronchoalveolar lymphocytosis in farmer's lung. Am Rev Respir Dis 1987; 135: 692-695.

6. Cormier Y, Bélanger J, Beaudoin J, Laviolette M, Beaudoin R, Hebert J. Abnormal bronchoalveolar lavage in asymptomatic dairy farmers: study of lymphocytes. Am Rev Respir Dis 1984; 130: 1046-1049.

7. Dakhama A, Israel-Assayag E, Cormier Y. Evidence of interleukin-2 sustained bronchoalveolar lymphocytosis and persistent interleukin-2 activity in farmer's lung disease. Am J Respir Crit Care Med 1994; 149 (2 pt 2): A83.

8. Grant IWB, Blyth W, Wardrop VE, Gordon RM, Pearson JCG, Mair A. Prevalence of farmer's lung in Scotland: a pilot survey. BMJ 1972; 1: 530-534.

9. Cormier Y, Tremblay GM, Fournier M, Israel-Assayag E. Long-term viral enhancement of lung response to Saccharopolyspora rectivirgula. Am J Respir Crit Care Med 1994; 149: 490-494.

10. Fogelmark B, Sjostran M, Rylander R. Pulmonary inflammation induced by repeated inhalations of beta-(1,3)-Dglucan and endotoxin. Int J Exp Pathol 1994; 75: 85-90

11. Terho EO, Koskimies S, Heinonen OP, Mantyjarvi R. HLA and farmer's lung. Eur J Respir Dis 1982; 63: 361-362.

12. Holt PG. Inhibitory activity of unstimulated alveolar macrophages on T-lymphocyte blastogenic response. Am Rev Respir Dis 1978; 118: 791-793.

13. McCombs CC, Michalski JP, Westerfield BT, Light RW. Human alveolar macrophages suppress the proliferative responses of peripheral blood lymphocytes. Chest 1982; 82: 266-271.

14. Spiteri MA, Poulter LW. Characterization of immune-inducer and suppressor macrophages from the normal human lung. Clin Exp Immunol 1991; 83: 157-162.

15. Roth DR, Golub SH. Human pulmonary macrophages utilize prostaglandins and transforming growth factor- $\beta$ to suppress lymphocyte activation. J Leuk Biol 1993; 53: $366-371$.

16. Swisher SG, Kiertscher SM, Golub SH, Holmes EC, Roth MD. Pulmonary macrophages suppress the proliferation and cytotoxicity of tumor- infiltrating lymphocytes. $\mathrm{Am}$ J Respir Cell Mol Biol 1993; 8: 486-492.

17. Steele MG, Herscowitz HB. Suppression of murine IgM, $\mathrm{IgG}, \operatorname{Ig} \mathrm{A}$ and $\mathrm{IgE}$ antibody responses by alveolar macrophages. Immunology 1993; 80: 62-67.

18. Wilkes DS, Yarbrough WC, Weissler JC. Human alveolar macrophages inhibits immunoglobulin production in response to direct B-cell mitogen. Am J Repir Cell Mol Biol 1993; 9: 141-147.

19. Strickland DH, Kees UR, Holt UR. Suppression of T-cell activation by pulmonary alveolar macrophages: dissociation of effects on TRC, IL-2R expression and proliferation. Eur Respir J 1994; 7: 2124-2130.

20. Upham JW, Strickland DH, Bilyk N, Robinson BW, Holt PG. Alveolar macrophages from humans and rodents selectively inhibit T-cell proliferation but permit T-cell activation and cytokine secretion. Immunology 1995; 84: 142-147.

21. Holt PG, McMenamin C. Defense against allergic sensitisation in the healthy lung: the role of inhalation tolerance. Clin Exp Allergy 1989; 19: 255-262.

22. Bilyk N, Holt PG. Inhibition of the immunosuppressive activity of resident pulmonary alveolar macrophages by granulocyte/macrophage colony-stimulating factor. J Exp Med 1993; 177: 1773-1777.

23. Thorens B, Mermod JJ, Vassalin P. Phagocytosis and inflammatory stimuli induce GM-CSF mRNA in macrophages through post-transcriptional regulation. Cell 1987; 48: 671-679.

24. Stoolman LM. Adhesion molecules involved in leukocyte recruitment and lymphocyte recirculation. Chest 1993; 103 (Suppl. 3): 79S-86S.

25. Denis M, Cormier Y, Tardif J, Ghadirian E, Laviolette M. Hypersensitivity pneumonitis: whole Micropolyspora faeni or antigens thereof stimulate the release of proinflammatory cytokines from macrophages. Am J Respir Cell Mol Biol 1991; 5: 198-203.

26. Tremblay GM, Israel-Assayag E, Sirois P, Cormier Y. Murine hypersensitivity pneumonitis: evidence for the role of eicosanoids and platelet-activating factor. Immunol Invest 1993; 22(5): 341-352.

27. Salvaggio JE, deShazo RD. Pathogenesis of hypersensitivity pneumonitis. Chest 1986; 89 (Suppl.): 190S-193S.

28. Chemlik F, Dolico G, Reed CE, Dickie H. Farmer's lung. J Allergy Clin Immunol 1975; 54: 180-188.

29. Rich EA, Cooper C, Toossi Z, et al. Requirement for cell-to-cell contact for the immunosuppressive activity of human alveolar macrophages. Am J Respir Cell Mol Biol 1991; 4: 287-294.

30. Trentin L, Migone N, Zambello R, et al. Mechanisms accounting for lymphocytic alveolitis in hypersensitivity pneumonitis. J Immunol 1990; 145: 2147-2154.

31. Schauble TL, Boom WH, Finegan CK, Rich EA. Characterization of suppressor function of human alveolar macrophages for T-lymphocyte responses to phytohemagglutinin: cellular selectivity, reversibility, and early events in Tcell activation. Am J Respir Cell Mol Biol 1993; 8: 89-97.

32. DeShazo RD, Banks DE, Diem JE, et al. Bronchoalveolar lavage cell-lymphocyte interactions in normal nonsmokers and smokers. Am Rev Respir Dis 1983; 127: 545-548.

33. Warren CPW. Extrinsic allergic alveolitis: a disease commoner in non-smokers. Thorax 1977; 32: 567-569.

34. Fireman E, Ben-Efraim S, Spinrad S, Topilsky M, Greif J. Suppressive mechanisms of alveolar macrophages in interstitial lung diseases: role of soluble factors and cell-to-cell contact. Eur Respir J 1993; 6: 956-964.

35. Gallagher RB, Guckian M, van Breda A, Oldum C, Fitzgerald MX, Feighery C. Altered immunological reactivity in alveolar macrophages from patients with sarcoidosis. Eur Respir J 1988; 1: 153-160.

36. Metzger Z, Hoffeld TJ, Oppenheim JJ. Macrophage-mediated suppression: evidence for participation of both hydrogen peroxide and prostaglandins in suppression of murine lymphocyte proliferation. J Immunol 1980; 124: 983-988.

37. Lauzon W, Lemaire I. Alveolar macrophage inhibition of lung-associated NK activity: involvement of prostaglandins and transforming growth factor- $\beta 1$. Exp Lung Res 1994; 20: 331-349.

38. Weissler JC, Mendelson C, Moya F, Yarbrough WC. Effect of interstitial lung disease macrophages on T-cell signal transduction. Am J Respir Crit Care Med 1994; 149: 191-196.

39. Marini M, Vittori E, Hollemborg J, Mattoli S. Expression of the potent inflammatory cytokines, granulocyte/ macrophage colony-stimulating factor and interleukin- 6 and interleukin-8, in bronchial epithelial cells of patients with asthma. J Allergy Clin Immunol 1992; 89: 1001-1009.

40. Wodnar-Filipowicz A, Heusser CH, Moroni C. Production of the hemopoietic growth factors GM-CSF and interleukin-3 by mast cells in response to IgE receptor-mediated activation. Nature 1989; 339: 150-151.

41. Laviolette M, Cormier Y, Loiseau A, Soler P, Leblanc P, Hance AJ. Bronchoalveolar mast cells in normal farmers 
and subjects with farmer's lung: diagnostic, prognostic, and physiologic significance. Am Rev Respir Dis 1991; 144: 855-859.

42. Chu HW, Cormier Y, Laviolette M. Immunocytochemical analysis of transbronchial biopsies in hypersensitivity pneumonitis. Am J Respir Crit Care Med 1995; 151 (4, 2 part 2): A607.

43. Pforte A, Gerth C, Voss A, et al. Proliferating alveolar macrophages in BAL and lung function changes in interstitial lung disease. Eur Respir J 1993; 6: 951-955.

44. Bitterman PB, Saltzman LE, Adelberg S, Ferrans VJ, Crystal RG. Alveolar macrophage replication: one mec- hanism for the expansion of mononuclear phagocyte population in the chronically inflamed lung. J Clin Invest 1984; 74: 460-469.

45. Metcalf D, Elliott MJ, Nicola NA. The excess numbers of peritoneal macrophages in granulocyte/macrophage colony-stimulating factor in transgenic mice are generated by local proliferation. J Exp Med 1992; 175: 877-884.

46. Pforte A, Brunner A, Gais P, et al. Increased levels of soluble serum interleukin-2 receptor in extrinsic allergic alveolitis correlate with interleukin-2 receptor expression on alveolar macrophages. J Allergy Clin Immunol 1994; 94: $1057-1064$. 